\title{
Un estudio comparativo del estrés percibido en estudiantes de ciencias administrativas y biológicas en tiempos de violencia ${ }^{1}$
}

\author{
Marie Leiner de la \\ Cabada \\ Universidad Autónoma \\ de Ciudad Juárez \\ Universidad de Texas Tech \\ marie.leiner@ttuhsc.edu
}

\section{Patricia Jiménez.}

Terrazas

Universidad Autónoma de Ciudad Juárez

pjimenez@uacj.mx

${ }^{1}$ En memoria del estudiante César Correa Ojeda, víctima de la violencia que prevalece en Ciudad Juárez.

\section{Resumen}

En estudios anteriores relacionados con estudiantes universitarios se considera que los principales factores que contribuyen al estrés son las exigencias del aprendizaje, el medio universitario per se, la búsqueda de empleo, las relaciones interpersonales y los desórdenes emocionales; sin embargo, existen pocos estudios que consideran los factores globales como el aumento desmedido y por un periodo largo de la violencia en el nivel local atribuida al crimen organizado. En este estudio se comparó el estrés percibido en tres grupos de estudiantes que cursan carreras en ciencias administrativas y biológicas, en tres periodos, incluyendo dos etapas donde la violencia en la ciudad ha sido considerada como extrema. Los resultados indican que existe un incremento de estrés en los estudiantes durante los periodos donde ha aumentado la violencia en el nivel local. El incremento es más evidente en las mujeres que en los hombres, independientemente de la carrera seleccionada. Es importante considerar el posible impacto que este incremento en el estrés puede tener en su formación y salud a corto y largo plazos.

Palabras clave: estrés, violencia global, socialización, comunicación en salud, estudiantes universitarios 


\title{
Stress perception of students of administrative and biological sciences in periods of violence: a comparative study
}

\begin{abstract}
In various studies carried out with university students, it has been considered that the primary factors that contribute to stress are: 1) the educational demands placed upon them; 2) the university environment itself; 3) the job seeking process; 4) their interpersonal relationships; and 5) emotional disorders. However, very few studies consider the probable impact that global stressors, such as an increase in local violence, will have on an individual. This study compares the perceived stress of three different university student groups from the biological and administrative sciences, including two groups measured at periods when city violence was considered extreme. The results indicate that there is an increase in the student's perceived stress in the periods when local violence has been deemed to be high. The increase is more evident in female students when compared to males, independent of the career path selected. It is important to consider the possible impact that an increase in stress might have on the student's professional formation, as well as on their short and long term health.
\end{abstract}

Keywords: Stress, global violence, socialization, health communication, college students.

\section{Introducción}

Los principales factores que contribuyen a causar estrés en los estudiantes en el ámbito universitario son las exigencias del aprendizaje, las presiones del medio universitario per se, la búsqueda de empleo, las relaciones interpersonales y los desórdenes emocionales (Shen, Kong y Hou, 2009). Estos factores generadores del estrés están íntimamente ligados a una época de intensos cambios sociales en la vida del estudiante; por ejemplo, deja de ser adolescente para convertirse en adulto y adquiere cierta independencia que generalmente está aunada a nuevas exigencias y responsabilidades (O’Donovan y Hughes, 2007).

Desde el punto de vista de la sociología, los factores que contribuyen a generar estrés en el estudiante universitario no son independientes, sino que están íntimamente ligados al medio en el que se desarrolla el individuo (Grant, Stewart y Mohr, 2009; Williams, Galanter, Dermatis y Schwartz, 2008). De tal forma que factores como la cultura, la organización social, económica, legal y política de la sociedad en la que el individuo se desenvuelve contribuyen a producir o atenuar los efectos del estrés de una forma directa, incluyendo tanto reacciones de pasividad como de agresividad (Parsons, 1954). 
Existen además factores ligados al medio en el que se desarrolla el individuo, que contribuyen a generar estrés con un carácter más global (Batley, Makhoul y Latif, 2008; Broussard, Myers y Meaux, 2008; Karabekiroglu et al., 2008; Rosenberg et al., 2008) y que pueden tener una presencia local, regional, estatal, etc., como es el caso de los efectos de una guerra, guerrilla, altibajos en la economía o la violencia generada a causa del llamado crimen organizado. Estos factores de estrés con carácter global tienen una característica distintiva que los ubica como factores de riesgo capaces de producir estrés crónico (Sagy, 2002) por su persistencia que habitualmente es de larga duración.

Los estudios sobre el impacto de estos factores de estrés con carácter global, como es el caso de la guerra o guerrilla, muestran una adversa influencia en el desempeño escolar del estudiante, no sólo por ser generadores de estrés, sino porque alteran las condiciones ideales en las que se enseña (Iacopino et al., 2001). Se considera que estos conflictos armados causan principalmente un deterioro en la salud mental del estudiante (Halligan, 2009; Hasanovic et al., 2009; Holtedahl, 2009; Panter-Brick et al., 2009; Sibai et al., 2009).

En el caso de los efectos de la violencia atribuida al crimen organizado, no se encontraron estudios que muestren el impacto que este tipo de violencia generalizada tiene como efecto generador de estrés en el estudiante universitario. Los efectos que este factor global puede provocar en los estudiantes son difíciles de producirse de forma artificial, por lo que su estudio es limitado; sin embargo, gracias a un estudio que empezó en 2004 con el objetivo de determinar el estrés percibido entre estudiantes universitarios en la Universidad Autónoma de Ciudad Juárez, y que se repitió en el primer y segundo semestres de 2009, generó información que permitió hacer un estudio comparativo sobre estrés percibido y el posible efecto de un factor global. La exposición a este factor global de estrés se produce en la vida cotidiana de los estudiantes universitarios, pues durante 2008 y 2009 los habitantes de Ciudad Juárez, Chihuahua, México, estuvieron expuestos a un aumento considerable de violencia atribuido al crimen organizado (AFP, 2009).

El objetivo de este estudio es hacer una comparación de estrés en estudiantes universitarios durante tres periodos 2004 (primer semestre escolar), 2009 (primer semestre) y 2009 (segundo semestre), considerando el posible efecto de un factor global generador de estrés a causa de la ola de violencia (2008-2009) atribuida al crimen organizado. En estudios relacionados con el estrés académico, se consideró que las carreras pertenecientes a ciencias biológicas tienen un efecto adicional de 
estrés en los estudiantes (Marty et al., 2005). Para este estudio comparativo se incluyeron tanto estudiantes que cursan carreras en ciencias biológicas como en ciencias administrativas (sociales).

Se propuso responder a las siguientes preguntas de investigación: ¿existen diferencias en el estrés percibido por estudiantes universitarios al comparar periodos?, ¿existen diferencias en el estrés percibido por los estudiantes universitarios al comparar género y periodos? y finalmente, ¿existen diferencias en el estrés percibido por los estudiantes universitarios al comparar a los que asisten a carreras en ciencias administrativas o biomédicas?

\section{Material y métodos}

\section{Tipo de estudio y población de estudio}

La recolección de datos se efectuó a partir de un estudio observacional, descriptivo, correlacional y transversal (transeccional) con recolección de datos incluyendo tres periodos: abril de 2004 (2004-1), abril de 2009 (2009-1) y octubre de 2009 (2009-2) sobre el estrés percibido por estudiantes universitarios que asisten a la Universidad Autónoma de Ciudad Juárez en los institutos de Ciencias Sociales y Administración (ICSA) y de Ciencias Biomédicas (ICB). Las áreas biomédicas incluyeron a estudiantes de las carreras de medicina, odontología o enfermería y en las carreras de tipo administrativo a estudiantes de administración, contaduría, turismo, entre otras.

Los datos incluidos en este estudio fueron recolectados a partir de una base de datos y fueron recopilados a partir de un muestreo probabilístico por racimos, carrera dentro del instituto, para determinar el número de alumnos necesarios por carrera; se incluyeron sólo a las licenciaturas calculando en función del total de alumnos por carrera y por instituto. Después de determinar la proporción de estudiantes por carrera, se completó el total de alumnos asistiendo a las aulas de clase y se recolectaron las respuestas de todos los que estuvieron presentes por cada carrera. Posteriormente, se presentó el protocolo a las autoridades universitarias y se solicitó la colaboración voluntaria de los alumnos durante clases con el permiso de sus maestros. 


\section{Instrumentos}

1. Para medir el estrés percibido se utilizó el cuestionario de estrés percibido (Cohen et al., 2005) y se empleó la versión que fue validada en español en México (Ramírez y Hernández, 2007).

2. Información demográfica; año de nacimiento, nivel de estudios (principiante, intermedio o avanzado), género (femenino o masculino), estado civil (soltero o casado), estar actualmente empleado (sí o no) e instituto (Instituto de Ciencias Sociales y Administración, ICSA, o Instituto de Ciencias Biomédicas, ICB). Respuestas a la pregunta sobre percepción de la violencia en la ciudad: ¿está enterado de la ola de violencia que existe en la ciudad? y sobre la economía: ¿se siente afectado por la economía que prevalece en la ciudad?

\section{Variables}

La variable dependiente para este estudio incluye el total de estrés percibido calculado a partir de las respuestas al cuestionario de Sheldon Cohen, Tony Kamarck y Robin Mermelstein (Cohen et al., 2005) que contiene 14 preguntas sobre el estrés percibido en el último mes y que incluyen una escala tipo Likert que varía a partir de $0=$ nunca a $4=$ con frecuencia. En el instrumento, los reactivos 4, 5, 6, 7, 9 y 10 se calculan invirtiendo los valores asignados a la escala. El total de estrés se obtiene sumando los valores de todas las preguntas.

\section{Variables independientes}

Estar enterado de la ola de violencia que existe en la ciudad Sentirse afectado por la economía que prevalece en la ciudad Periodo de medición, estar laborando, género, edad, área de estudio, estado civil, empleo, Nivel de estudios (principiante, intermedio, avanzado). Edad agrupada en 17-21, 22-26, 27-32, 33-40 y > 40 años.

Información general sobre factores globales de violencia durante los periodos:

- 2004. La violencia que prevalecía en la ciudad incluía como tema prioritario el asesinato de mujeres.

- 2009-1. La violencia que prevalecía en la ciudad se atribuía al crimen organizado. La recolección de información ocurrió poco después de la llegada del ejército a Ciudad Juárez. 
- 2009-2. La violencia que prevalecía en la ciudad continuaba siendo atribuida al crimen organizado.

\section{Análisis}

Se utilizaron estadísticas descriptivas para analizar medias y desviaciones estándar de las diferentes variables numéricas y frecuencias para las variables categóricas. Para verificar las asociaciones entre las variables del estudio se utilizaron diferentes pruebas estadísticas según el nivel de medición de las variables, tales como la t de estudiante, ANOVA y el coeficiente de correlación Pearson. Para identificar los mejores predictores de la variable de estrés percibido se utilizó la técnica de regresión lineal múltiple por el método "paso por paso" (stepwise). Se compararon los resultados de la percepción del estrés con muestras de estudiantes en México y en otros países.

\section{Características de la muestra.}

Se recolectaron un total de 3114 cuestionarios con un total de 2454 (78.8\%) respondidos en forma completa. La distribución por género se detalla en el cuadro 1 , y por instituto en el cuadro 2. Debido a que todos los estudiantes respondieron estar al tanto de la violencia y afectados por la economía en todos los periodos, se omite el uso de las variables en todo el estudio. El promedio de edad de toda la muestra fue de 23 años con una SD = 4.6. La media de edades en 2004-1 fue de 22.7 con $\mathrm{SD}=4.6$ en el 2009-1 de 23.5 SD = 4.4 y en el 2009-2 de $22.6 \mathrm{SD}=4.7$.

\section{Cuadro 1}

Distribución por genero 2004-2009

\begin{tabular}{l|r|r|r}
\hline \multirow{3}{*}{2004 Licenciatura } & \multicolumn{3}{|c}{ Género } \\
\cline { 2 - 4 } 2009 Licenciatura Primer Semestre & 320 & 435 & \multicolumn{1}{c}{ Total } \\
\cline { 2 - 4 } 2009 Licenciatura Segundo Semestre & $42.4 \%$ & $57.6 \%$ & $100.0 \%$ \\
& 381 & 567 & 948 \\
Total & $40.2 \%$ & $59.8 \%$ & $100.0 \%$ \\
& 257 & 494 & 751 \\
& $34.2 \%$ & $65.8 \%$ & $100.0 \%$ \\
& 958 & 1496 & 2454 \\
& $39.0 \%$ & $61.0 \%$ & $100.0 \%$ \\
\hline
\end{tabular}

$\chi^{2}=11.4 \mathrm{df}=2 \mathrm{p}=.003$ 


\section{Cuadro 2}

Distribución de participantes por instituto 2004-2009

\begin{tabular}{l|r|r|r}
\hline & \multicolumn{3}{|c}{ Instituto } \\
\hline Periodo & \multicolumn{1}{|c|}{ ICSA } & \multicolumn{1}{c|}{ ICB } & \multicolumn{1}{c}{ Total } \\
\cline { 2 - 4 } 2004 Licenciatura & 400 & 355 & 755 \\
& $53.0 \%$ & $47.0 \%$ & $100.0 \%$ \\
2009 Licenciatura Primer Semestre & 212 & 736 & 948 \\
& $22.4 \%$ & $77.6 \%$ & $100.0 \%$ \\
2009 Licenciatura Segundo Semestre & 300 & 451 & 751 \\
& $39.9 \%$ & $60.1 \%$ & $100.0 \%$ \\
Total & 912 & 1542 & 2454 \\
& $39.0 \%$ & $61.0 \%$ & $100.0 \%$ \\
\hline
\end{tabular}

$\chi^{2}=172.3 \mathrm{df}=2 \mathrm{p}<.001$

\section{Resultados}

Existe una diferencia significativa en la edad de los participantes de 2009-1 al comparar la media de edades (prueba de ANOVA y Bonferroni para promedios), se detectó la diferencia al comparar la muestra de 2004-1 con la de 2009-2. Lo mismo ocurre con la distribución de participantes por género donde se observa una mayor cantidad de mujeres en 2009 al compararlas con 2004. La distribución por estado civil se detalla en el siguiente cuadro 3 .

\section{Cuadro 3}

Distribución de participantes por estado civil 2004-2009

\begin{tabular}{l|r|r|r}
\hline & \multicolumn{2}{|c|}{ Estado civil } & \\
\hline Periodo & Soltero & Casado/en pareja & Total \\
\cline { 2 - 4 } 2004 Licenciatura & 620 & 118 & 738 \\
& $84.0 \%$ & $16.0 \%$ & $100.0 \%$ \\
\multirow{3}{*}{2009 Licenciatura Primer Semestre } & 798 & 147 & 945 \\
& $84.4 \%$ & $15.6 \%$ & $100.0 \%$ \\
Total & 633 & 114 & 747 \\
& $84.7 \%$ & $15.3 \%$ & $100.0 \%$ \\
& 2051 & 379 & 2430 \\
& $84.4 \%$ & $15.6 \%$ & $100.0 \%$ \\
\hline
\end{tabular}

$\chi^{2}=0.15 \mathrm{df}=2 \mathrm{p}=0.093$ Diferencia con el total fue debido a valores perdidos 
No existe diferencia significativa en el estado civil de los participantes en los tres periodos. Las estadísticas de tendencia central y dispersión del promedio de estrés percibido por género, por instituto y por periodo se detallan en el cuadro 4.

\section{Cuadro 4}

Promedios de estrés percibido por género, instituto y periodo 2004-2009

\begin{tabular}{|c|c|c|c|c|c|}
\hline Grupo & Instituto & Género & Promedio & $\mathbf{N}$ & Desviación \\
\hline \multirow{6}{*}{$\begin{array}{l}2004 \\
\text { Licenciatura }\end{array}$} & \multirow{3}{*}{ ICB } & Hombre & 23.58 & 180 & 7.65 \\
\hline & & Mujer & 26.31 & 220 & 6.92 \\
\hline & & Total & 25.08 & 400 & 7.40 \\
\hline & \multirow{3}{*}{ ICSA } & Hombre & 24.41 & 140 & 6.77 \\
\hline & & Mujer & 25.25 & 215 & 7.10 \\
\hline & & Total & 24.92 & 355 & 6.97 \\
\hline \multirow{6}{*}{$\begin{array}{l}2009 \\
\text { Licenciatura } \\
\text { Primer Semestre }\end{array}$} & \multirow{3}{*}{ ICB } & Hombre & 23.49 & 83 & 7.52 \\
\hline & & Mujer & 26.62 & 129 & 7.70 \\
\hline & & Total & 25.40 & 212 & 7.75 \\
\hline & \multirow{3}{*}{ ICSA } & Hombre & 23.80 & 298 & 7.25 \\
\hline & & Mujer & 25.66 & 438 & 7.13 \\
\hline & & Total & 24.90 & 736 & 7.23 \\
\hline \multirow{6}{*}{$\begin{array}{l}2009 \\
\text { Licenciatura } \\
\text { Segundo } \\
\text { Semestre }\end{array}$} & \multirow{3}{*}{ ICB } & Hombre & 24.84 & 88 & 8.72 \\
\hline & & Mujer & 26.74 & 212 & 7.91 \\
\hline & & Total & 26.18 & 300 & 8.20 \\
\hline & \multirow{3}{*}{ ICSA } & Hombre & 23.94 & 169 & 7.28 \\
\hline & & Mujer & 26.87 & 282 & 8.05 \\
\hline & & Total & 25.77 & 451 & 7.89 \\
\hline \multirow{9}{*}{ Total } & \multirow{3}{*}{ ICB } & Hombre & 23.87 & 351 & 7.90 \\
\hline & & Mujer & 26.54 & 561 & 7.47 \\
\hline & & Total & 25.52 & 912 & 7.74 \\
\hline & \multirow{3}{*}{ ICSA } & Hombre & 23.98 & 607 & 7.15 \\
\hline & & Mujer & 25.93 & 935 & 7.43 \\
\hline & & Total & 25.16 & 1542 & 7.38 \\
\hline & & Hombre & 23.94 & 958 & 7.43 \\
\hline & & Mujer & 26.16 & 1496 & 7.45 \\
\hline & & Total & 25.29 & 2454 & 7.52 \\
\hline
\end{tabular}

En cuanto a la relación de las variables independientes con el estrés percibido prueba Pearson de correlación, se encontró lo siguiente: género, edad agrupada, estado civil y periodo tienen una asociación significativa; en cambio el nivel de estudios, empleo, periodo e instituto no tiene una asociación significativa. 


\section{Cuadro 5}

\section{Correlaciones entre variables dependientes e independientes}

\begin{tabular}{l|l|l|l|l|l|l|l}
\hline & $\begin{array}{c}\text { Total } \\
\text { estrés }\end{array}$ & Grupo & Instituto & Género & Trabaja & $\begin{array}{c}\text { Estado } \\
\text { civil }\end{array}$ & $\begin{array}{c}\text { Edad } \\
\text { codificada }\end{array}$ \\
\hline Pearson Correlación & & .053 & -.023 & .144 & .028 & -.057 & -.094 \\
Sig. (2-tailed) & & $.008^{* *}$ & .257 & $.000^{* *}$ & .169 & $.005^{* *}$ & $.000^{* *}$ \\
$\mathrm{~N}$ & 2454 & 2454 & 2454 & 2454 & 2452 & 2430 & 2443 \\
\hline
\end{tabular}

** Correlación es significante al nivel 0.01 (2-tailed).

Estrés intragénero por periodos e instituto. Existe una diferencia significativa entre el estrés percibido por los hombres con respecto a las mujeres en todos los periodos. Al comparar el estrés de los hombres por periodos no se encontró una diferencia significativa. En el caso de las mujeres se encontró una diferencia significativa al comparar los periodos del 2004 con el 2009-1 y con el periodo 2009-2. Al comparar el estrés percibido por periodo, instituto y género se encontró la diferencia en el caso de las mujeres asistiendo a ICSA al comparar el periodo de 2004 con el 2009-1 y 2009-2. Todas las demás comparaciones fueron no-significativas.

\section{Cuadro 6}

\section{Diferencias de estrés intragénero por periodo e instituto}

\begin{tabular}{|c|c|c|c|}
\hline Periodo & 2004 Licenciatura & $\begin{array}{l}2009 \text { Licenciatura } \\
\text { Primer semestre }\end{array}$ & $\begin{array}{l}2009 \text { Licenciatura } \\
\text { Segundo semestre }\end{array}$ \\
\hline \multirow[t]{2}{*}{$\begin{array}{l}\text { Hombres } \\
\text { vs. Mujeres }\end{array}$} & $\begin{array}{l}\text { Dif }=-1.85 \\
(\mathrm{t}=-3.5 \mathrm{df}=753 \mathrm{p} \\
<.001 * *)\end{array}$ & $\begin{array}{l}\text { Dif }=-2.1 \\
(\mathrm{t}=-2.1 \mathrm{df}=946 \mathrm{p}<.001)^{* *}\end{array}$ & $\begin{array}{l}\text { Dif }=-2.6 \\
(\mathrm{t}=-4.2 \mathrm{df}=749 \mathrm{p}<.001)^{* *}\end{array}$ \\
\hline & $\begin{array}{l}2004 \text { Licenciatura } v s . \\
2009 \text { Licenciatura } \\
\text { Primer semestre }\end{array}$ & $\begin{array}{l}2004 \text { Licenciatura vs. } \\
2009 \text { Licenciatura } \\
\text { Segundo Semestre }\end{array}$ & $\begin{array}{l}2009 \text { Licenciatura Primer } \\
\text { Semestre vs. } 2009 \\
\text { Licenciatura Segundo } \\
\text { Semestre }\end{array}$ \\
\hline Hombres & $*$ & $*$ & $*$ \\
\hline \multirow[t]{2}{*}{ Mujeres } & $*$ & $\begin{array}{l}\text { Dif }=-1.0 \\
(\mathrm{t}=-2.1 \mathrm{df}=944 \mathrm{p}=0.04 * *)\end{array}$ & $\begin{array}{l}\text { Dif }=-0.9 \\
(\mathrm{t}=-2.0 \mathrm{df}=1059 \mathrm{p} \\
\left.=0.045^{* *}\right)\end{array}$ \\
\hline & $\begin{array}{l}2004 \text { Licenciatura vs. } \\
2009 \text { Licenciatura } \\
\text { Primer semestre }\end{array}$ & $\begin{array}{l}2004 \text { Licenciatura vs. } \\
2009 \text { Licenciatura } \\
\text { Segundo semestre }\end{array}$ & $\begin{array}{l}\text { 2009 Licenciatura Primer } \\
\text { Semestre vs. } 2009 \\
\text { Licenciatura Segundo } \\
\text { semestre }\end{array}$ \\
\hline $\begin{array}{l}\text { Hombres } \\
\text { vs. Mujeres } \\
\text { ICB }\end{array}$ & $*$ & $*$ & (2) \\
\hline $\begin{array}{l}\text { Hombres } \\
\text { vs. Mujeres } \\
\text { ICSA }\end{array}$ & $*$ & $\begin{array}{l}\text { Dif }=-1.6 \\
(\mathrm{t}=-2.3 \mathrm{df}=495 \mathrm{p}=0.02 * *)\end{array}$ & $\begin{array}{l}\text { Dif }=-1.2 \\
(\mathrm{t}=-2.1 \mathrm{df}=718 \mathrm{p}=0.04 * *)\end{array}$ \\
\hline
\end{tabular}

* No es significativo ** Significativo 


\section{Análisis gráfico}

El análisis gráfico del estrés muestra la diferencia durante los tres periodos por género (ver figura 1).

Figura 1

Diferencias de estrés intragénero por periodo

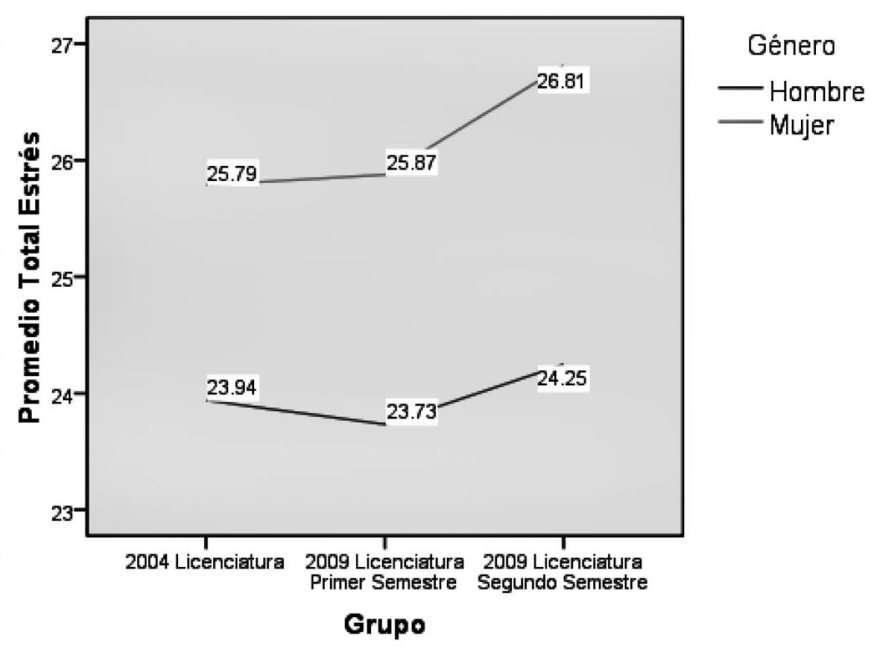

En esta gráfica se puede observar que las mujeres declararon tener un estrés percibido mayor al de los hombres en todos los periodos con una tendencia al aumento a partir del 2004.

La representación gráfica (ver figura 2) muestra que el estrés de los hombres del Instituto ICB es más alto que el de los hombres del Instituto de Ciencias Sociales, con una diferencia que no es significativa como se mostró anteriormente. En el caso de las mujeres el estrés percibido durante el 2004 y el 2009-1 es más alto en el caso de las estudiantes de ciencias biomédicas. En 2009 en los dos periodos, el estrés de las estudiantes de ciencias administrativas es muy similar al de las estudiantes de ciencias biológicas. 
Figura 2

Diferencias intragénero por instituto y periodo

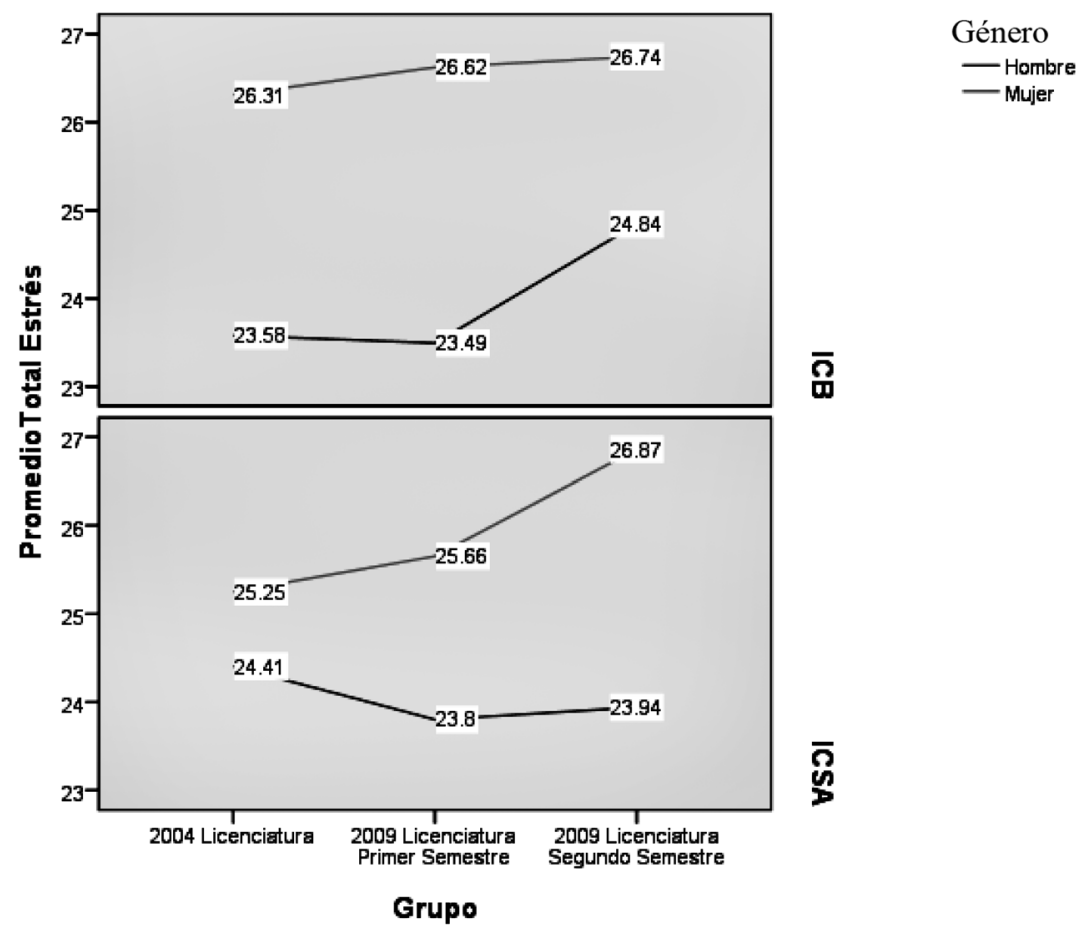

\section{Variables predictoras del estrés}

En el análisis de regresión múltiple (modelo stepwise), los resultados del modelo final se presentan en el cuadro 7. Para la muestra total, las variables predictoras fueron género, periodo y edad agrupada. Los resultados del análisis de varianza ANOVA fueron significativos [F (10.3) $\mathrm{p}<0.001]$, con un coeficiente de determinación (ajustado $\mathrm{R}^{2}=0.26$ ), indicando que estas variables explican el $26 \%$ del total de la variación. El análisis de Durbin Watson fue de 1.9, lo que demuestra que los residuales fueron independientes. Los resultados indican que de todas las variables independientes que se incluyeron en el modelo sólo algunas explican la variación incluyendo el periodo en el que se hizo la medición, género y la edad. Todas las demás variables fueron descartadas. 


\section{Cuadro 7}

\section{Variables predictoras del estrés - Modelo de regresión lineal múltiple}

\begin{tabular}{c|c|c|c|c}
\hline & $\begin{array}{c}\text { Variables sig- } \\
\text { nificativas usando } \\
\text { stepwise }\end{array}$ & $\mathbf{b}$ & Error típico & P \\
\hline Modelo & Edad & -0.08 & 0.04 & $0.03 *$ \\
& Género & 2.02 & 0.32 & $<.001^{*}$ \\
& Periodo & 0.28 & 0.13 & $0.03 *$ \\
\hline
\end{tabular}

$\mathrm{F}=10.3 \mathrm{p}<.001$ Durbin Watson $1.9 \mathrm{df}=\mathrm{R}^{2}=0.26 *$ Significativo

\section{Comparativo con otros estudios}

Se compararon los resultados obtenidos en este estudio con los de estudiantes en México y en otros países utilizando el mismo instrumento de medición.

\section{Cuadro 8}

\section{Comparativo con estudiantes de diversas carreras usando la versión de 14 reactivos}

\begin{tabular}{c|c|c|c|c}
\hline País/Estudios & & $\mathbf{2 0 0 4}$ & $\mathbf{2 0 0 9 - 1}$ & $\mathbf{2 0 0 9 - 2}$ \\
\hline México & \multicolumn{4}{|c}{ Promedio(SD) } \\
Estudiantes & 365 & 755 & 948 & 751 \\
de psicología & $21.9(7.3)$ & $25.0(7.2)^{*}$ & $25.0(7.3)^{*}$ & $25.9(8.0)^{*}$ \\
Japon/Reino Unido & \multicolumn{4}{|c|}{ Promedio(SD) } \\
Enfermeras & $1214 / 222$ & 755 & 948 & 751 \\
Farmacéuticos & $27.6(8.4)$ & $25.0(7.2)^{* *}$ & $25.0(7.3)^{* *}$ & $25.9(8.0)^{* *}$ \\
España & \multicolumn{4}{|c|}{ Promedio(SD) } \\
Ciencias administrativas & 208 & 755 & 948 & 751 \\
y Biomedicas & $29.2(8.2)$ & $25.0(7.2)^{* *}$ & $25.0(7.3)^{* *}$ & $25.9(8.0)^{* *}$ \\
Portugal & \multicolumn{4}{|c|}{ Promedio(SD) } \\
Ciencias administrativas & 239 & 755 & 948 & 751 \\
y Biomedicas & $26.8(7.2)$ & $25.0(7.2)^{* *}$ & $25.0(7.3)^{* *}$ & $25.9(8.0)^{* *}$ \\
Estados Unidos & \multicolumn{4}{|c|}{ Promedio(SD) } \\
Doctorado en enfermería & $22.9(7.9)$ & $25.0(7.2)^{*}$ & $25.0(7.3)^{*}$ & $25.9(8.0)^{*}$ \\
\hline
\end{tabular}

* Significativo UACJ mayor promedio

** Significativo UACJ menor promedio 
Al comparar el promedio del estrés de la muestra de estudiantes en este estudio, se observa un promedio mayor de estrés percibido cuando se compara con el obtenido por estudiantes de psicología en México y estudiantes de doctorado de enfermería en los Estados Unidos. Menores promedios se observan al comparar con estudiantes de enfermería y farmacia en Japón y el Reino Unido al igual que en España y Portugal con una muestra similar a la de este estudio en ciencias administrativas y biomédicas.

\section{Discusión}

Por segundo año consecutivo Ciudad Juárez se ha considerado como la ciudad más violenta del mundo de acuerdo con la organización civil mexicana: Consejo Ciudadano para la Seguridad Pública (CCSP) (AFP, 2009). La posibilidad de un efecto global generador de estrés en los estudiantes universitarios a causa de la exposición a la violencia atribuida al crimen organizado se observó al comparar los tres periodos. El estrés reportado por los estudiantes es más alto en los periodos 2009-1 y 2009-2 cuando se comparan con el periodo 2004-1, especialmente en el caso de las mujeres; en cambio, en los hombres el reporte indica un promedio más alto en el periodo 2009-2 cuando se compara con el semestre 2004-1.

En general el estrés reportado por las mujeres es siempre más alto y significante que el de los hombres en todos los periodos. Adicionalmente, el estrés percibido reportado por las mujeres parece tener una tendencia hacia el aumento cuando se comparan los periodos del 2009 con 2004. Se observa una disminución numérica en el promedio de estrés reportado en hombres y mujeres en el 2009-1 al compararlos con el 2009-2, que coincide con la llegada del ejército a Ciudad Juárez, Chihuahua, México.

Al comparar el estrés percibido de este estudio con el único estudio que se encontró en estudiantes universitarios en México —usando el mismo instrumento- la muestra de Ciudad Juárez mostró promedios más altos al comparar cualquiera de los periodos. No se observó en este estudio, como se ha reportado con anterioridad, una diferencia entre el estrés reportado por estudiantes de ciencias biomédicas al compararlos con los de ciencias administrativas (Marty et al., 2005). Incluso, en el caso de las mujeres que cursan carreras en ciencias administrativas se observa un promedio más alto y significativo al de las estudiantes de ciencias biológicas. 
El factor global causado por la violencia atribuida al crimen organizado parece mostrar un efecto generador de estrés, que puede tener importantes implicaciones en la salud y comportamiento de los estudiantes. Para entender su posible impacto, es necesario diferenciar las condiciones que prevalecen para generar el estrés considerado como agudo del crónico.

La respuesta que los seres humanos dan al estrés se relaciona como una reacción natural, e incluso útil, pues actúa como protección en caso de enfrentar un peligro porque desencadena una respuesta inmediata de protección como es el caso de necesitar huir del peligro (Mawson, 2005). Este tipo de estrés prepara al cuerpo para pelear o huir ante la inminencia del peligro. Es también generado como respuesta a emociones como las que se producen cuando los atletas participan en una competencia, o cuando en presentaciones de artistas les da un impulso adicional de energía o creatividad. Desde el punto de vista fisiológico permite el bombeo de sangre a los músculos mayores, aumenta el ritmo del latido del corazón y la presión sanguínea (Gibbons, Dempster y Moutray, 2008). Al pasar el evento que generó el estrés con posibles cambios fisiológicos temporales, el organismo regresa a su estado normal, sin que se observen efectos duraderos o con carácter permanente. Existen, sin embargo, otros eventos que originan estrés con un carácter agudo y/o crónico, que producen efectos de mayor duración y que pueden causar problemas de carácter fisiológico, emocional o conductual.

En el caso del estrés agudo, que es el resultado de un evento intenso, la duración del evento es corta; es decir, pasa con rapidez, como ejemplo se puede mencionar los cambios de rutina. La persona reacciona al cambio, pero vuelve a la tranquilidad dentro de un lapso relativamente breve. En el caso del estrés crónico, el individuo confronta cambios constantes de rutina que ocurren con periodicidad y durante periodos de larga duración. El estrés crónico, al afectar al organismo durante periodos largos, se considera que tiene efectos a nivel orgánico que pueden ser perjudiciales a la salud (Martin y Martin, 2002; Sagy, 2002).

La duración de los eventos estresantes juega un importante factor en la respuesta del individuo con un impacto en las respuestas emocionales, fisiológicas y de comportamiento que pueden ser tanto de corta duración como permanentes (Landis, 2007; McEwen, 1998). Esto incluye eventos que persisten por periodos largos como es el caso de la enfermedad seria de un ser querido con algunas excepciones, incluyendo aquellos eventos de corta duración que son muy traumáticos, como es el caso de un asalto sexual o agresión seria (Baum, Cohen y Hall, 1993). 
El estrés crónico es el que más se relaciona con enfermedades de origen fisiológico y psicosociales. Entre las de origen fisiológico se encuentran las enfermedades crónicas como las de origen cardiovascular o cáncer (Cohen et al., 2005). La exposición al estrés de forma continua parece causar una aceleración en la formación de placas en las arterias que potencialmente pueden contribuir a complicaciones de la enfermedad conocida como arteriosclerosis (Bosch et al., 2003) y comprometer el sistema inmunológico (Segerstrom, 2007; Segerstrom, Schipper y Greenberg, 2008). En pruebas de laboratorio, usando ratones, se ha encontrado que ante el efecto continuo del estrés, la ansiedad juega un importante rol en la inflamación y degeneración de neuronas predisponiendo a infecciones recurrentes e inflamación crónica (Bouayed et al., 2007; Rammal et al., 2008).

En relación con las enfermedades psicosociales, existe una mayor cantidad de investigaciones sobre el efecto del estrés que han sido desarrolladas por psicólogos (Biegler, Chaoul y Cohen, 2009; Glynn et al., 2009; Kendall y Terry, 2009; Zohar et al., 2009), con estudios que muestran tanto los efectos a corto y largo plazos (Goudriaan, et al., 2009; Kumar et al., 2009; Tharion, Parthasarathy y Neelakan$\tan , 2009)$.

En el caso de los estudiantes, la depresión crónica, las respuestas agresivas como resultado del estrés, el desinterés por la vida escolar y los problemas de comportamiento han sido documentados como resultado del efecto de otros factores globales como es el caso de la guerra, guerrilla o la violencia comunitaria (Abdel-Khalek, 2006; Abdullatif, 1995; Halligan, 2009; Jellinek, Murphy y Burns, 1986; NuttmanShwartz y Dekel, 2009; Oswalt y Silberg, 1995; Pacifico y Lester, 1994).

En el caso del factor global de la violencia, que se muestra en este estudio, el impacto del efecto continuo y por un periodo largo no se puede determinar a partir de los resultados de este estudio; sin embargo, es posible inferir que existe un estrés adicional con características de cronicidad, pues el problema de la violencia atribuida al crimen organizado no ha terminado. El estudiante universitario en Ciudad Juárez, Chihuahua, continuará expuesto a este factor global de estrés por un periodo de mayor duración.

Los estudiantes universitarios se encuentran en un proceso de formación importante para su vida profesional y para la sociedad. Las presiones inherentes a la vida universitaria deben ser superadas por el estudiante, sin estragos a su salud física y emocional con la finalidad de que se favorezcan las mejores condiciones para que 
puedan cumplir con su obligación como profesionales y como miembros de la sociedad. Si bien es cierto que no se sabe el efecto profesional o social que el estrés adicional que enfrentan estos estudiantes causará a largo plazo, no se puede dejar de lado que la violencia generalizada, y la ausencia de un sistema de seguridad y justicia reflejado en la impunidad que prevalece en la ciudad, está siendo también parte de su formación. Por esto, es importante considerar que aunque el factor global de la violencia generalizada atribuida al crimen organizado puede terminar eventualmente, las consecuencias que se germinan tanto en las mentes como en el organismo de los estudiantes pueden no terminar al mismo tiempo.

Este estudio tiene ciertas limitaciones que incluyen el que, debido a que las carreras tienen diferente número de estudiantes, existen diferencias significativas entre el número de participantes por género y por instituto, por lo que no se asociaron la posibilidad de ser una víctima o testigo de violencia con la variable de estrés para este estudio. Es posible que existan variaciones entre el nivel de exposición a la violencia que pueden tener un impacto en el estrés percibido; de tal forma que algunos estudiantes pueden estar más afectados que otros. Adicionalmente, debido a que los profesores pueden hacer exámenes a su propia discreción, es posible que haya una variabilidad adicional que no se detectó, al recopilar los datos de aquellos participantes que estaban teniendo un examen antes o después de responder. Asimismo, la situación económica que ha prevalecido en forma global en 2009 pudo haber influido en el estrés percibido por los participantes, aunque no se consideró que esta variable global haya afectado sustancialmente los resultados porque todos los estudiantes declararon estar afectados por la economía en los tres periodos.

Finalmente, en este estudio se observa que no todos los estudiantes responden de la misma forma, posiblemente por la existencia de mecanismos de defensa; sin embargo, ya que esta variable no se consideró en el estudio, no es posible reportar mas información sobre las diferencias reportadas. A pesar de estas limitaciones, las tendencias son sustancialmente claras, el tamaño de la muestra es considerable porque indica que los resultados que se obtuvieron posiblemente no sean el resultado de la casualidad, sino de las variables independientes consideradas en este estudio. 


\section{Referencias}

Abdel-Khalek, A. M. (2006). Comparison of death anxiety in two groups tested during and after (1986 and 1991) the Lebanese Civil War. Psychol Rep, 98(3), 712-714.

Abdullatif, H. I. (1995). Prevalence of depression among middle-school Kuwaiti students following the Iraqi invasion. Psychol Rep, 77(2), 643-649.

AFP. (2009). Ciudad Juárez la ciudad más violenta del mundo. Proceso.

Batley, N. J., J. Makhoul y S. A. Latif (2008). War as a positive medical educational experience. Med Educ, 42(12), 1166-1171.

Baum, A., L. Cohen y M. Hall (1993). Control and intrusive memories as possible determinants of chronic stress. Psychosom Med, 55(3), 274-286.

Biegler, K. A., M. A. Chaoul y L. Cohen (2009). Cancer, cognitive impairment, and meditation. Acta Oncol, 48(1), 18-26.

Bosch, J. A., G. Berntson, J. T. Cacioppo, F. S. Dhabhar y P. T.Marucha (2003). Acute stress evokes selective mobilization of $\mathrm{T}$ cells that differ in chemokine receptor expression: a potential pathway linking immunologic reactivity to cardiovascular disease. Brain Behav Immun, 17(4), 251-259.

Bouayed, J., H. Rammal, C. Younos y R. Soulimani (2007). Positive correlation between peripheral blood granulocyte oxidative status and level of anxiety in mice. Eur J Pharmacol, 564(1-3), 146-149.

Broussard, L., R. Myers y J. Meaux (2008). The impact of hurricanes Katrina and Rita on Louisiana school nurses. J Sch Nurs, 24(2), 78-82.

Cohen, A. L., F. Rivara, E. K. Marcuse, H. McPhillips y R. Davis (2005). Are language barriers associated with serious medical events in hospitalized pediatric patients? Pediatrics, 116(3), 575-579.

Gibbons, C., M. Dempster y M. Moutray (2008). Stress and eustress in nursing students. J Adv Nurs, 61(3), 282-290. 
Glynn, K., H. Maclean, T. Forte y M. Cohen (2009). The association between role overload and women's mental health. J Womens Health (Larchmt), 18(2), 217-223.

Goudriaan, A. E., W. S. Slutske, J. L. Krull y K. J. Sher, (2009). Longitudinal patterns of gambling activities and associated risk factors in college students. Addiction, 104(7), 1219-1232.

Grant, V. V., S. H. Stewart y C. D Mohr, (2009). Coping-anxiety and coping-depression motives predict different daily mood-drinking relationships. Psychol Addict Behav, 23(2), 226-237.

Halligan, F. R. (2009). Youth and trauma: terror, war, murder, incest, rape, and suicide. J Relig Health, 48(3), 342-352.

Hasanovic, M., S. Srabovic, M. Rasidovic, M. Sehovic, E. Hasanbasic, J. Husanovic et al. (2009). Psychosocial assistance to students with posttraumatic stress disorder in primary and secondary schools in post-war Bosnia Herzegovina. Psychiatr Danub, 21(4), 463-473.

Holtedahl, K. (2009). Peace work: some lessons from medicine and biology. Med Confl Surviv, 25(1), 65-77.

Iacopino, V., M. W. Frank, H. M. Bauer, A. S. Keller, S. L. Fink, D. Ford et al. (2001). A population-based assessment of human rights abuses committed against ethnic Albanian refugees from Kosovo. Am J Public Health, 91(12), 2013-2018.

Jellinek, M. S., J. M. Murphy y B. J. Burns (1986). Brief psychosocial screening in outpatient pediatric practice. J Pediatr, 109(2), 371-378.

Karabekiroglu, K., S. Akbas, G. N. Tasdemir y M. N. Karakurt (2008). Post-traumatic stress symptoms in adolescents after two murders in a school: a controlled follow-up study. Int J Psychiatry Med, 38(4), 407-424.

Kendall, E. y D. Terry (2009). Predicting emotional well-being following traumatic brain injury: a test of mediated and moderated models. Soc Sci Med, 69(6), 947-954. 
Kumar, S., R. J. Dagli, A. Mathur, M. Jain, D. Prabu y S. Kulkarni (2009). Perceived sources of stress amongst Indian dental students. Eur J Dent Educ, 13(1), 39-45.

Landis, C. (2007). Why the inflammatory response is important to the cardiac surgical patient. J Extra Corpor Technol, 39(4), 281-284.

Martin, T. J. y J. S. Martin (2002). Special issues and concerns for the high schooland college-aged athletes. Pediatr Clin North Am, 49(3), 533-552.

Marty, C., M. Lavín, M. Figueroa, C, D. L. d. 1. y C.C. M. (2005). Prevalencia de estrés en estudiantes del área de la salud de la Universidad de los Andes y su relación con enfermedades infecciosas. Rev Chil Neuro-Psiquiat, 43 (1), 25-32.

Mawson, A. R. (2005). Understanding mass panic and other collective responses to threat and disaster. Psychiatry, 68(2), 95-113.

McEwen, B. S. (1998). Protective and damaging effects of stress mediators. $N$ Engl J Med, 338(3), 171-179.

Nuttman-Shwartz, O. y R. Dekel (2009). Ways of coping and sense of belonging in the face of a continuous threat. J Trauma Stress, 22(6), 667-670.

O’Donovan, A. y B. Hughes (2007). Social support and loneliness in college students: effects on pulse pressure reactivity to acute stress. Int J Adolesc Med Health, 19(4), 523-528.

Oswalt, R. y K. Silberg (1995). Self-perceived traumatic stress in college: a survey. Psychol Rep, 77(3 Pt 1), 985-986.

Pacifico, C., y D. Lester (1994). Attitudes toward war in adolescents. Psychol Rep, 75(1 Pt 2), 394.

Panter-Brick, C., M. Eggerman, V. Gonzalez y S. Safdar (2009). Violence, suffering, and mental health in Afghanistan: a school-based survey. Lancet, 374(9692), 807-816. 
Parsons, T. (1954). Certain Primary Sources and Patterns of Aggression in the Social Structure of the Western World: Essays in Sociological Theory (Vol. 1). Glencoe, Ill: Free Press.

Ramírez, M. T. y R. L. Hernández (2007). Factor structure of the Perceived Stress Scale (PSS) in a sample from Mexico. Span J Psychol, 10(1), 199-206.

Rammal, H., J. Bouayed, C. Younos y R. Soulimani (2008). Evidence that oxidative stress is linked to anxiety-related behaviour in mice. Brain Behav Immun, 22(8), 1156-1159.

Rosenberg, A., R. G. Heimberg, Z.Solomon y L. Levin (2008). Investigation of exposure-symptom relationships in a context of recurrent violence. $J$ Anxiety Disord, 22(3), 416-428.

Sagy, S. (2002). Moderating factors explaining stress reactions: comparing chronic-without-acute-stress and chronic-with-acute-stress situations. J Psychol, 136(4), 407-419.

Segerstrom, S. C. (2007). Stress, Energy, y Immunity: An Ecological View. Curr Dir Psychol Sci, 16(6), 326-330.

Segerstrom, S. C., L. J. Schipper y R. N. Greenberg (2008). Caregiving, repetitive thought, and immune response to vaccination in older adults. Brain Behav Immun, 22(5), 744-752.

Shen, L., H. Kong, H y X. Hou (2009). Prevalence of irritable bowel syndrome and its relationship with psychological stress status in Chinese university students. J Gastroenterol Hepatol, 24(12), 1885-1890.

Sibai, T., R. A. Tohme, H. A. Beydoun, N. Kanaan y A. M. Sibai (2009). Violent behavior among adolescents in post-war Lebanon: the role of personal factors and correlation with other problem behaviors. J Public Health (Oxf), 31(1), 39-46.

Tharion, E., S. Parthasarathy y N. Neelakantan (2009). Short-term heart rate variability measures in students during examinations. Natl Med J India, 22(2), 63-66. 
Williams, C. B., M. Galanter, H. Dermatis y V. Schwartz (2008). The importance of hopelessness among university students seeking psychiatric counseling. Psychiatr Q, 79(4), 311-319.

Zohar, J., R. Sonnino, A.Juven-Wetzler y H. Cohen (2009). Can posttraumatic stress disorder be prevented? CNS Spectr, 14(1 Suppl 1), 44-51. 
